\title{
Study of the system responsivity to measure the blackbody's temperature by optical pyrometry from $1200 \mathrm{~K}$ to $1570 \mathrm{~K}$
}

\author{
Saif Abbane*, Zahra Ben Achour, and Oualid Touayar \\ University of Carthage, National Institute of Applied Sciences and Technology, INSAT BP 676 Centre Urbain Nord, \\ 1080 Tunis Cedex, Tunisia
}

Received: 9 October 2016 / Accepted: 1 December 2016

\begin{abstract}
This work presents a method that has been recently adopted in our laboratory to determine the temperatures of blackbody sources in the range of 1200-1570 K. The system uses a Double Monochromator System (DMS) based on a grating and a prism as dispersion elements. The detection element was a silicon photodiode (Si-MMA), over which the spectral range from $800 \mathrm{~nm}$ to $900 \mathrm{~nm}$ has been used. Between the blackbody source and the DMS was placed an optical system consists of two convergent lenses. The system responsivity " $G$ " was determined by the transmission factor of the optical system and the transmission factor of the DMS and the photodiode responsivity. The obtained results showed that the relative uncertainty of the system responsivity " $G$ " varied from $0.3 \%$ to $1.12 \%$. This in turn resulted in a corresponding uncertainty in temperature of about $2.2 \mathrm{~K}$ and $4.5 \mathrm{~K}(k=1)$ over the evaluated temperature range. Although this uncertainty level was significantly high compared to those obtained by many other national metrology institutes, it was considered as a step forward in our laboratory to measure high temperatures.
\end{abstract}

Keywords: temperature / pyrometry / system responsivity / detector / optical transmission

\section{Introduction}

On the basis of The mise en pratique for the definition of the kelvin (MeP-K) [1-3] and what have been shown by Yamada et al. in 1999 that is possible to use metal-carbon binary eutectic alloys as potential fixed points above the freezing point of copper $\left(T_{\mathrm{Cu}}=1357.77 \mathrm{~K}\right)$ to determine the thermodynamic temperature [4,5]. This has been followed by the Consultative Committee for Thermometry (CCT) to develop and improve the thermal and radiometric performance of metal (carbide)-carbon ( $\mathrm{M}(\mathrm{C})-\mathrm{C})$ fixed points [6,7]. The efficacy of this method is part of a study that was made as well in the context the European Metrology Research Programme (EMRP) joint project 'Implementing the new kelvin' (Ink) [8] was focused on preparing the temperature metrology community for a comprehensive and effective redefinition of the kelvin [9].

A second method is to use a filter radiometer with a known spectral response and defined measurement geometry can determine the thermodynamic temperature of blackbody sources $[5,10]$.

The temperature references of the International Temperature Scale ITS-90 below the freezing point of copper $(1357.77 \mathrm{~K})$ are based on various fixed points

* Corresponding author: saif .aben@gmail.com
( $\mathrm{Cu}, \mathrm{Au}, \mathrm{Ag}, \mathrm{Zn}, \mathrm{Al}$, etc.). Above the copper reference point the optical pyrometry based on Planck's radiation law using monochromatic radiation is necessary [11].

The radiance is the selected reference parameter used to materialize the thermodynamic temperature unit within the range of high temperatures $(1234.93-3000 \mathrm{~K})$. In practice, the spectral radiance is used to express the radiance contained in an elementary wavelength window $\mathrm{d} \lambda$ including the wavelength $\lambda$. This radiance is expressed in $\mathrm{W} \mathrm{m}^{-3} \mathrm{sr}^{-1}$.

For the blackbody, the spectral radiance density within the range of $\lambda$ and $\lambda+\lambda d$ is expressed in terms of Planck's radiation law connecting the temperature $T$ and the wavelength $\lambda$ of the emitted radiation. The Planck's law of radiation is used in the definition of the thermodynamic temperature unit [12].

The spectral radiance ratio of two blackbodies can be traced back to the temperature in the ITS 90 scale [11].

The spectral radiance density, in a given direction, at a given point of a surface, is directly defined by the spectral radiant flux transmitted by an elementary beam passing through the given point and propagating in the solid angle $\mathrm{d} \Omega$ containing the given direction [13].

The blackbody temperature accuracy depends heavily on the flux measurement and dimensional quantities accuracies. For the flux measurements, we used silicon 


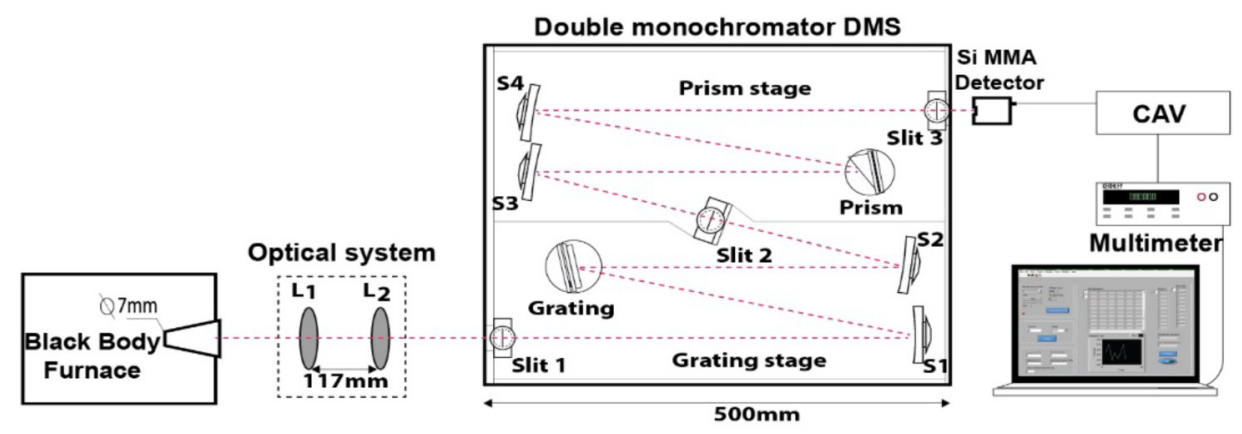

$\mathbf{L}_{1}, \mathbf{L}_{2}$ : Convergent lens

$S_{1}, S_{2}, S_{3}, S_{4}$ : Spherical mirror

CAV : current-voltage converter

Fig. 1. Schematic diagram of experimental bench.

photodiodes. The determination of the dimensional values, with high accuracy, essentially depends on the quality of the bench set up materializing the solid angle and the emitting surface [14].

The materialization of the solid angle is done through the implementation of an optical system based on convergent lenses and diaphragms. This system has different reflections, absorptions and transmissions levels which are difficult to evaluate, so annexes benches are required.

As Planck's radiation law depends strongly on the monochromatic wavelength, we used a device based on double monochromator (DMS) incorporating a grating and a prism allowing respectively to disperse and to refine the selected wavelength. Despite the utilization of that device, the imperfect knowledge of the chosen wavelength and the use of the integrated optical introduce errors and uncertainties on the temperatures determination.

Consequently, the combination of the double monochromator and the optical system, together define the system responsivity " $G$ " which involves the optical and dimensional effects. This system responsivity provides the correspondence between the flux received by the photodiode and the flux emitted by the source. Thus, the received flux would be the image of the emitted one via this system responsivity " $G$ " characterizing the experimental bench. The determination of " $G$ " is essential in order to determine the unknown temperature $T_{x}$ of any blackbody furnace exposed to our system.

This paper describes the method used to determine the system responsivity " $G$ " with the required accuracy and this within the spectral range from $800 \mathrm{~nm}$ to $900 \mathrm{~nm}$ and in the temperature range between $1200 \mathrm{~K}$ and $1570 \mathrm{~K}$. The choice of these ranges is dictated by the silicon photodiode operating range. Therefore, the measuring range of temperature on one hand, covers the last three fixed points of the ITS $90(\mathrm{Ag}, \mathrm{Au}$ and $\mathrm{Cu})$ and in the other hand, is limited by the maximum threshold of our blackbody furnace CNHT.

Two methods are applicable for determining $G$, either through a calibrated fixed-point blackbody or by using a blackbody furnace whose temperatures are checked using a transfer pyrometer. In our case, we choose the last method to achieve our goal.

\section{Experimental set up}

The bench presented in Figure 1 is intended to calibrate optical pyrometers. It is composed mainly of a Lanthanum Chromite blackbody furnace CNHT [15] at high temperatures which is equipped with a variable temperature blackbody. The dynamic temperature of this blackbody covers

873.15-1773.15K. The blackbody furnace provides a 7-mm diameter emission diaphragm and with a current regulated source with a stability of $10^{-5}$.

In all cases, the Lanthanum Chromite cavities requiring following re-verification on a standard blackbody fixed points in the entire spectral band established by the ITS 90 in the range from $600 \mathrm{~nm}$ to $1000 \mathrm{~nm} \mathrm{[12].}$

The cavity bottom of the furnace is imaged by the spectroradiometer which consisted of convergent lenses $\mathrm{L}_{1}$ and $\mathrm{L}_{2}$ having focal distances of $4 \mathrm{~cm}$ and $8 \mathrm{~cm}$, respectively. By the help of these two lenses the cavity bottom of the furnace can be focused on the entrance slit of the double monochromator with a diameter about $25 \mathrm{~mm}$.

The flux enters the double monochromator (DMS) through the rectangular inlet slit (slit 1) with a height of $2 \mathrm{~cm}$. This double monochromator is composed by a grating 300 lines/mm blazed at $1000 \mathrm{~nm}$ and a silica's prism [16]. After undergoing some reflections by spherical mirrors $\mathrm{S}_{1}$, $\mathrm{S}_{2}, \mathrm{~S}_{3}$ and $\mathrm{S}_{4}$ located in the two stages of the double monochromator (grating stage and prism stage), the diffracted light falls on the silicon detector active area placed directly behind the exit slit of the monochromator. The width of the batch of light falling on the silicon detector is $3 \mathrm{~mm}$ which is less than the photodiode active area dimension estimated about $10 \mathrm{~mm}$ allowing thus flux measurement.

The photodiode is used along with a current to voltage operational amplifier, which converts the light induced photocurrent to a potential difference measured across amplifier resistance. The voltage is then measured by a digital multimeter, a numerical code controls the grating and prism motors used to tune the wavelength of the blackbody source. 


\section{Theory calculation}

\subsection{System responsivity " $G$ " determination}

The determination of the system responsivity " $G$ " that characterizes the experimental bench is accomplished via the expression of Planck's radiation law that considers the energetic flux of the emitting surface, the sensor responsivity, the temperature $T_{\mathrm{CNHT}}$ measured by a transfer pyrometer and the wavelength $\lambda$ of the blackbody emitted radiation. The transfer pyrometer we used is a Heitronics KT 19 II type. This is an Infrared Radiation Pyrometer based on pyroelectric detector with a response spectral from $2 \mu \mathrm{m}$ to $4.5 \mu \mathrm{m}$; its accuracy is about $\pm 0.5^{\circ} \mathrm{C}$ plus $0.7 \%$ of the temperature difference between the housing containing the measuring instruments and the object to be measured [17].

By applying Planck's radiation law for a blackbody, the total flux emitted over the hemisphere per unit area is the emittance. We generally consider $\mathrm{d} \phi$ the elementary radiant flux emitted by an area element $\mathrm{d} S$ in the various directions where it can radiate and we divide the flux by $\mathrm{d} S$ $[14,18]$.

The total radiant emittance based on the elementary flux is defined as [14]:

$$
M^{0}=\frac{\mathrm{d} \phi}{\mathrm{d} S}\left(\mathrm{~W} / \mathrm{m}^{2}\right)
$$

Furthermore, the spectral flux emitted according to the spectral radiant emittance is:

$$
\phi_{\lambda_{e}}=M_{T_{\mathrm{CNHT}}}^{0}(\lambda) \cdot S_{e} .
$$

The radiate power by the silicon detector is written:

$$
\phi_{\lambda_{r}}=\frac{I}{S_{\lambda}}=\frac{V}{S_{\lambda} R}
$$

So

$$
\phi_{\lambda_{r}}=F_{S_{e} \rightarrow S_{r}} \cdot \tau_{\left(\lambda, T_{a}\right)} \cdot \phi_{\lambda_{e}}
$$

Then

$$
\phi_{\lambda_{r}}=F_{S_{e} \rightarrow S_{r}} \cdot \tau_{\left(\lambda, T_{a}\right)} \cdot S_{e} \cdot M_{T_{\mathrm{CNHT}}}^{0}(\lambda)
$$

Thus

$$
F_{S_{e} \rightarrow S_{r}} \cdot \tau_{\left(\lambda, T_{a}\right)}=\frac{\phi_{\lambda_{r}}}{M_{T_{\mathrm{CNHT}}}^{0}(\lambda) S_{e}} .
$$

The combination between the two equations (3) and (5) gives:

$$
\begin{gathered}
\frac{V}{R \cdot S_{\lambda}}=S_{e} \cdot F_{S_{e} \rightarrow S_{r}} \cdot \tau_{\left(\lambda, T_{a}\right)} \frac{C_{1} \cdot \lambda^{-5}}{\exp \left(C_{2} /\left(\lambda \cdot T_{\mathrm{CNHT}}\right)\right)-1}, \\
G=F_{S_{e} \rightarrow S_{r}} \cdot \tau_{\left(\lambda, T_{a}\right)}=\frac{V}{R S_{\lambda} S_{e} C_{1} \lambda^{-5}}\left(\exp \left(\frac{C_{2}}{\lambda T_{\mathrm{CNHT}}}\right)-1\right) .
\end{gathered}
$$

$G$ is the system responsivity of our experimental bench. With:

$M^{0}$ : total radiant emittance; $C_{1}=3.74 \times 10^{-16} \mathrm{~W} \mathrm{~m}^{-3}$; $C_{2}=1.4388 \times 10^{-12} \mathrm{~m} \mathrm{~K} ; M^{0}=\pi L^{0} \mathrm{~W} \mathrm{~m}^{-3} ; F_{S_{e} \rightarrow S_{r}}$ : DMS transmission factor; $\tau_{\left(\lambda, T_{a}\right)}$ : optical transmission factor; $F_{S_{e} \rightarrow S_{r}} \cdot \tau_{\left(\lambda, T_{a}\right)}$ : system responsivity; $S_{e}$ : emitter surface; $S_{r}$ : receiver surface; $\phi_{\lambda_{e}}$ : spectral flux emitted; $\phi_{\lambda_{r}}$ : radiate power; $S_{\lambda}$ : photodiode spectral responsivity; $V$ : voltage delivered by the photodiode; $I$ : photocurrent; $R$ : amplification resistance of the converter current/voltage device.

\subsection{Temperature determination and measurement uncertainty}

As we said from the beginning, the objective of our work is to determine the temperature of any blackbody with its uncertainty through the use of the system responsivity.

For a radiant blackbody with an unknown temperature $T_{x}$, placed at the entrance of given the optical bench, Through the use of Planck's radiation law $T_{x}$ is expressed as follows:

$$
T_{x}=\frac{C_{2}}{\lambda} \frac{1}{\operatorname{Ln}\left(1+\left(R S_{\lambda} S_{e} G C_{1} \lambda^{-5}\right) / V\right)} .
$$

The parameters involved have been previously defined. The uncertainties propagation law associated to the temperature $T_{x}$ gives the following relative uncertainty $u_{T_{x}} / T_{x}$ following:

$$
\begin{gathered}
\frac{u_{T_{x}}}{T_{x}}=\sqrt{\left(\frac{\partial T_{x}}{\partial R}\right)^{2}\left(\frac{u_{R}}{R}\right)^{2}+\left(\frac{\partial T_{x}}{\partial S_{\lambda}}\right)^{2}\left(\frac{u_{S_{\lambda}}}{S_{\lambda}}\right)^{2}+\left(\frac{\partial T_{x}}{\partial V}\right)^{2}\left(\frac{u_{V}}{V}\right)^{2}} \\
+\left(\frac{\partial T_{x}}{\partial \lambda}\right)^{2}\left(\frac{u_{\lambda}}{\lambda}\right)^{2}+\left(\frac{\partial T_{x}}{\partial G}\right)^{2}\left(\frac{u_{G}}{G}\right)^{2} \cdot
\end{gathered}
$$

With:

$$
\begin{aligned}
\frac{\partial T_{x}}{\partial R} & =\frac{G S_{\lambda} S_{e} C_{1} C_{2}}{\left(\lambda R S_{\lambda} S_{e} G C_{1}+\lambda^{6} V\right) L n^{2}\left(\left(\left(R S_{\lambda} S_{e} G C_{1}\right) / \lambda^{5} V\right)+1\right)} \\
& =\frac{G S_{\lambda} S_{e} C_{1}}{\left(R S_{\lambda} S_{e} G C_{1}+\lambda^{5} V\right) \operatorname{Ln}\left(\left(\left(R S_{\lambda} S_{e} G C_{1}\right) / \lambda^{5} V\right)+1\right)} \cdot T_{x},
\end{aligned}
$$

$$
\begin{aligned}
\frac{\partial T_{x}}{\partial G} & =\frac{R S_{\lambda} S_{e} C_{1} C_{2}}{\left(\lambda R S_{\lambda} S_{e} G C_{1}+\lambda^{6} V\right) L n^{2}\left(\left(\left(R S_{\lambda} S_{e} G C_{1}\right) / \lambda^{5} V\right)+1\right)} \\
& =\frac{R S_{\lambda} S_{e} C_{1}}{\left(R S_{\lambda} S_{e} G C_{1}+\lambda^{5} V\right) \operatorname{Ln}\left(\left(\left(R S_{\lambda} S_{e} G C_{1}\right) / \lambda^{5} V\right)+1\right)} \cdot T_{x},
\end{aligned}
$$

$$
\begin{aligned}
\frac{\partial T_{x}}{\partial V} & =\frac{G R S_{\lambda} S_{e} C_{1} C_{2}}{\lambda V\left(R S_{\lambda} S_{e} G C_{1}+\lambda^{5} V\right) L n^{2}\left(\left(\left(R S_{\lambda} S_{e} G C_{1}\right) / \lambda^{5} V\right)+1\right)} \\
& =\frac{G R S_{\lambda} S_{e} C_{1}}{V\left(R S_{\lambda} S_{e} G C_{1}+\lambda^{5} V\right) \operatorname{Ln}\left(\left(\left(R S_{\lambda} S_{e} G C_{1}\right) / \lambda^{5} V\right)+1\right)} \cdot T_{x},
\end{aligned}
$$




\section{Transmission Factor of the optical system for different temperatures}

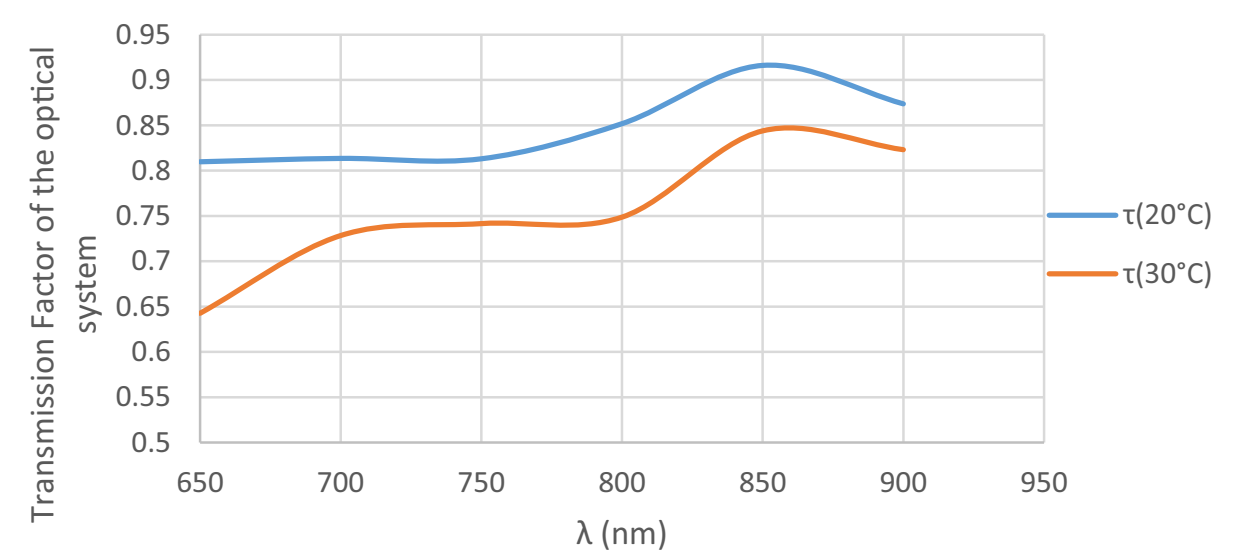

Fig. 2. Transmission factor of the optical system.

$$
\begin{aligned}
\frac{\partial T_{x}}{\partial \lambda}= & \frac{5 G R S_{\lambda} S_{e} C_{1} C_{2}}{\lambda^{7} V\left(\left(\left(R S_{\lambda} S_{e} G C_{1}\right) / \lambda^{5} V\right)+1\right)} \\
& L n^{2}\left(\left(\left(R S_{\lambda} S_{e} G C_{1}\right) / \lambda^{5} V\right)+1\right) \\
- & \frac{C_{2}}{\lambda^{2} \operatorname{Ln}\left(\left(\left(R S_{\lambda} S_{e} G C_{1}\right) / \lambda^{5} V\right)+1\right)} \\
= & \left(\frac{5 G R S_{\lambda} S_{e} C_{1}}{\lambda^{6} V\left(\left(\left(R S_{\lambda} S_{e} G C_{1}\right) / \lambda^{5} V\right)+1\right)}\right. \\
& \left.\operatorname{Ln}\left(\left(\left(R S_{\lambda} S_{e} G C_{1}\right) / \lambda^{5} V\right)+1\right)-\frac{1}{\lambda}\right) \cdot T_{x} .
\end{aligned}
$$

\subsection{Systematic effects: room temperature effect}

Blackbody sources operated at high temperature emits considerable thermal load. It has been observed that when the blackbody operates the temperature range of interest $(1223.15 \mathrm{~K}$ and $1423.15 \mathrm{~K})$ temperature of the optical system, which composed from the two lenses, changes from $20^{\circ} \mathrm{C}$ to $30^{\circ} \mathrm{C}$. This in turns would affect the spectroradiometer system, thus, its responsivity. In order for this effect to be evaluated, the transmittance of the two lenses has been measured at variable temperatures from $20^{\circ} \mathrm{C}$ to $30^{\circ} \mathrm{C}$ and the change of transmittance at different wavelengths has been evaluated. This has been carried out by using a quartz halogen lamp directly placed in front of the monochromator; however, the mirrors were placed behind the monochromator and just before the detection element.

Two spherical mirrors having a $99 \%$ reflectance, reflect the radiation delivered from the DMS, to the optical system. The transmission of the optical system essentially depends on the nature of the glasses, the wavelength $\lambda$ and the ambient temperature $T_{a}$ in which the system is arranged.

The detector used is a silicon photodiode. In our study, we are interested to the wavelengths effects between $650 \mathrm{~nm}$ and $900 \mathrm{~nm}$ on optical system transmission for the temperatures of $20^{\circ} \mathrm{C}$ and $30^{\circ} \mathrm{C}$. The ambient temperature $T_{a}$ is measured by a thermocouple $\mathrm{k}$ type placed close to the optical system.

The transmission factor $\tau$ for a given wavelength is defined as the ratio between the detector signal with the optical system and the total signal without the optical system

Figure 2 summarizes the experimental results obtained from the determination of the optical system transmission factor for both ambient temperatures $20^{\circ} \mathrm{C}$ and $30^{\circ} \mathrm{C}$.

These values obtained of $\tau_{(\lambda, \text { Ta) }}$ will be used to adjust the system responsivities " $G$ " according to the ambient temperatures corresponding to blackbodies' temperatures.

\section{Results and discussion}

\subsection{Si-MMA detector spectral responsivity}

The calibration of the (Si-MMA) detector used in our spectroradiometer, was done through the use of a standard trap detector calibrated traceable to the primary laboratory LNE - CNAM (France).

The estimated relative uncertainty of the absolute spectral responsivity determination is about $3 \times 10^{-3}$ to a $1 \sigma$ confidence level. This uncertainty takes into account the uncertainty of the standard Si-CNAM, The repeatability, the reproducibility of measurements, the current-voltage converter and the used voltmeter calibration.

Figure 3 shows the shape of the absolute spectral responsivity of the Si-MMA photodiode used in our bench.

In the range between $800 \mathrm{~nm}$ and $900 \mathrm{~nm}$, the absolute spectral responsivity of the used silicon photodiode in our experimental bench is at its maximum value while keeping a linear variation and increases according to the wavelength and achieves the peak near $950 \mathrm{~nm}$. To use the bench with the best signal stability, we will choose the range between $800 \mathrm{~nm}$ and $900 \mathrm{~nm}$. This choice will therefore determine the range of our experimental bench. 


\section{Photodiode spectral responsivity}

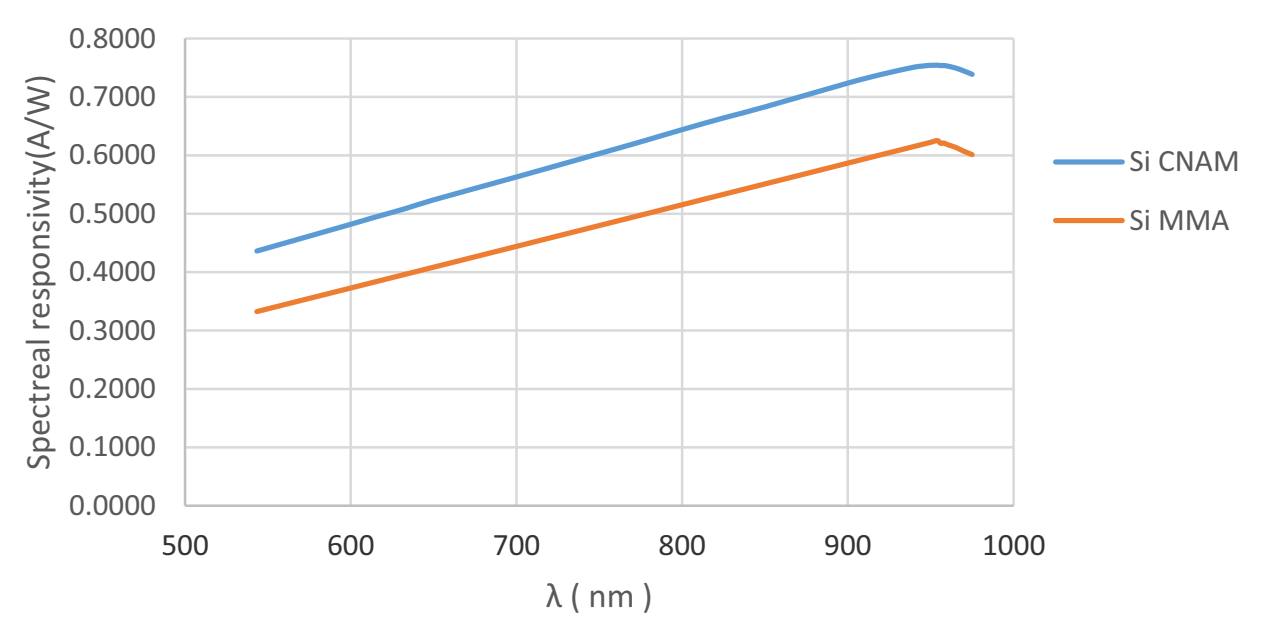

Fig. 3. Spectral responsivities of Si-MMA and Si-CNAM detectors.

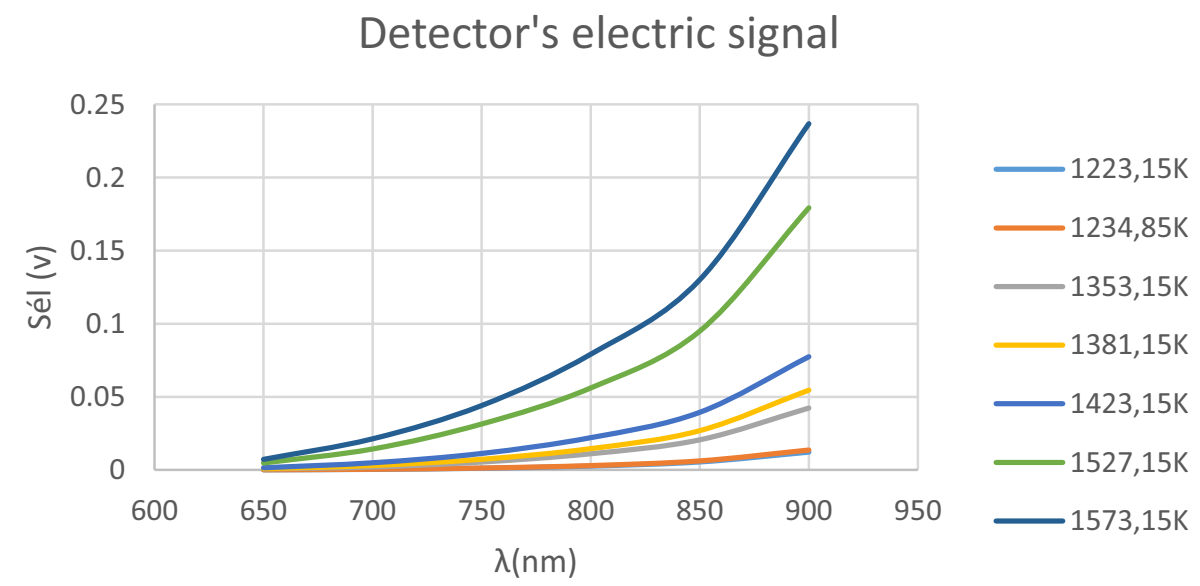

Fig. 4. Electric responses of Si-MMA detector for different temperatures of the blackbody furnace CNHT.

\subsection{The electric signal}

The signal delivered by the Si-MMA detector is the response of the experimental bench for various temperatures ranging from $1223.15 \mathrm{~K}$ to $1573.15 \mathrm{~K}$ and in the wavelength range from $650 \mathrm{~nm}$ to $900 \mathrm{~nm}$ integrating all the parameters involved (Fig. 4).

We noticed that the shape of the Si-MMA detector responses according to the wavelength was similar to Planck's radiation law applied to the wavelength range from $650 \mathrm{~nm}$ to $900 \mathrm{~nm}$. These responses are of a major importance in the determination of the unknown temperature $T_{x}$ of any blackbody.

\subsection{System responsivity measuring, $G\left(\lambda, T_{\mathrm{CNHT}}, T_{a}\right)$}

Having identified the responsivity of the system, as shown earlier, it has then been used to determine the temperature.
In our case, we consider for each wavelength, $\bar{G}$ the average of eight (08) $G_{i}$ values corresponding to eight (08) different temperatures from $1223.15 \mathrm{~K}$ to $1573.15 \mathrm{~K}$.

Table 1 summarizes the values of $\bar{G}$ for all the used wavelengths. For the uncertainty estimation we took the worst case and we estimated the uncertainty $u \bar{G}$ as the maximum deviation recorded for the $\bar{G}$ values $G_{\text {and }}$ for each wavelength.

Figure 5 shows the variation of the system responsivity " $G$ " of the experimental bench depending on the wavelength, for various temperatures.

We noticed that in the range between $800 \mathrm{~nm}$ and $900 \mathrm{~nm}$, the errors introduced by the system responsivity on the determination of the temperature $T_{x}$ are less than the errors between $650 \mathrm{~nm}$ and $800 \mathrm{~nm}$. This is due to the linear variation of the spectral responsivity of the silicon photodiode in this range. 
Table 1. System responsivity average for each wavelength.

\begin{tabular}{|c|c|c|c|c|c|c|c|}
\hline$\lambda(\mathrm{nm})$ & $\frac{u_{V}}{V}$ & $\frac{u_{\lambda}}{\lambda}$ & $\frac{u_{S_{\lambda}}}{S_{\lambda}}$ & $\frac{u_{R}}{R}$ & $\frac{u_{T_{\mathrm{CNHT}}}}{T_{\mathrm{CNHT}}}$ & $\bar{G}$ & $\frac{{ }^{u} \bar{G}}{\bar{G}}$ \\
\hline 650 & 0.00001 & 0.005 & 0.003 & 0.00001 & 0.007 & $9.4093 \mathrm{E}-14$ & 0.0334 \\
\hline 700 & 0.00001 & 0.005 & 0.003 & 0.00001 & 0.007 & $1.4606 \mathrm{E}-13$ & 0.0313 \\
\hline 750 & 0.00001 & 0.005 & 0.003 & 0.00001 & 0.007 & $1.7532 \mathrm{E}-13$ & 0.0275 \\
\hline 800 & 0.00001 & 0.005 & 0.003 & 0.00001 & 0.007 & $1.9565 \mathrm{E}-13$ & 0.0032 \\
\hline 850 & 0.00001 & 0.005 & 0.003 & 0.00001 & 0.007 & $2.3353 \mathrm{E}-13$ & 0.0069 \\
\hline 900 & 0.00001 & 0.005 & 0.003 & 0.00001 & 0.007 & $2.8743 \mathrm{E}-13$ & 0.0112 \\
\hline
\end{tabular}

Table 2. Uncertainties associated to different temperatures for three wavelengths selected.

\begin{tabular}{llllllllll}
\hline$\lambda(\mathrm{nm})$ & $\frac{u_{V}}{V}$ & $\frac{u_{\bar{G}}}{\bar{G}}$ & $\frac{u_{\lambda}}{\lambda}$ & $\frac{u_{S_{\lambda}}}{S_{\lambda}}$ & $\frac{u_{R}}{R}$ & $\frac{T_{1223.15}(\mathrm{~K})}{u_{T_{1223.15}}(\mathrm{~K})}$ & & $\frac{T_{1353.15}(\mathrm{~K})}{u_{T_{1353.15}}(\mathrm{~K})}$ & $\frac{T_{1573.15}(\mathrm{~K})}{u_{T_{1573.15}}(\mathrm{~K})}$ \\
\hline 800 & 0.00001 & 0.0032 & 0.005 & 0.003 & 0.00001 & 2.13 & 2.61 & 3.58 \\
850 & 0.00001 & 0.0069 & 0.005 & 0.003 & 0.00001 & 2.32 & 2.84 & 3.88 \\
900 & 0.00001 & 0.0112 & 0.005 & 0.003 & 0.00001 & 2.60 & 3.18 & 4.34 \\
\hline
\end{tabular}

\section{System responsivity variation}

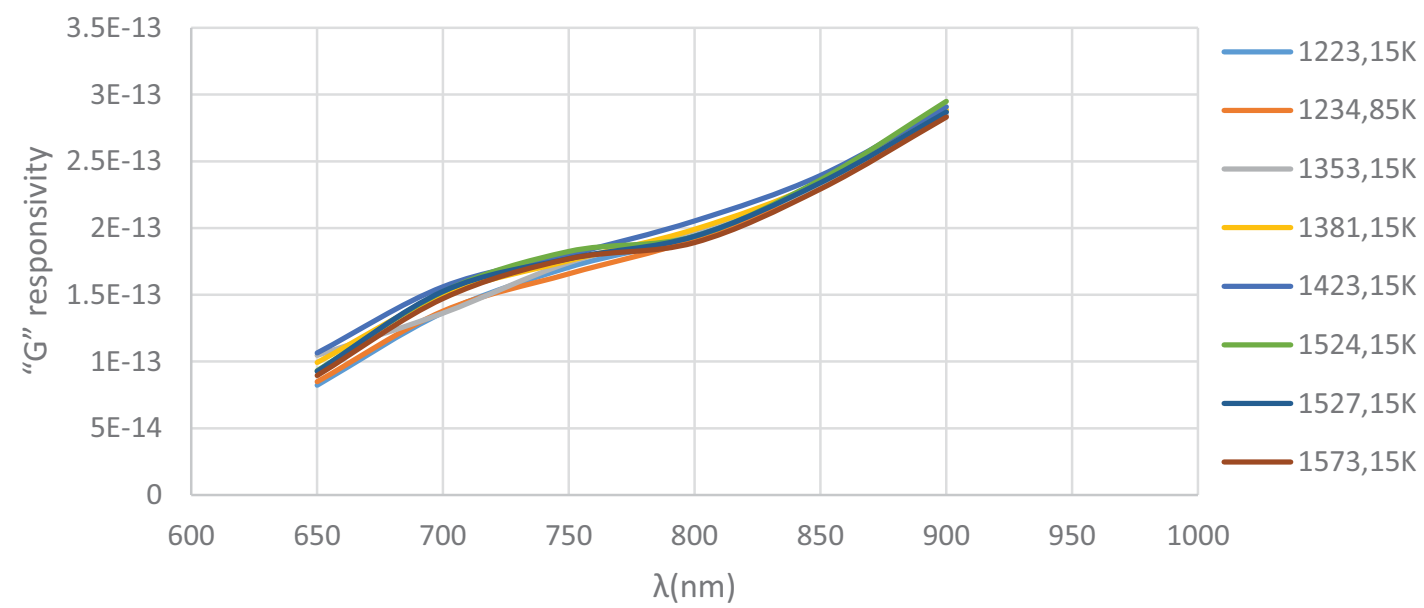

Fig. 5. System responsivities for each temperature.

This approach allows us to consider that the relative uncertainty associated to $\bar{G}$ vary from $0.3 \%$ to $1.12 \%$. These levels of uncertainty have a major importance in the uncertainties estimation of the blackbodies' unknown temperatures $T_{x}$.

\section{Discussion}

Using the equations (9) and (10) and considering the $\bar{G}$ values (Tab. 1) and their corresponding uncertainties, we are hence able to determine any temperature $T_{x}$ between $1223.15 \mathrm{~K}$ and $1573.15 \mathrm{~K}$ and estimate their associated uncertainty.

Table 2 gives practical examples of specific temperatures and their uncertainties for three selected wavelengths.
For a given wavelength, the uncertainty of the temperature increases according to the temperature. Also for a given temperature, the uncertainty $u_{T_{x}}$ increases according to the wavelength. This is predictable because in the expression of the uncertainty associated to $T_{x}$ (Eq. (10)), the only predominant uncertainties are those related to the average of the system responsivities $\bar{G}$ and the wavelength.

For our pyrometer bench, we should select the wavelength of $800 \mathrm{~nm}$ to obtain the lowest uncertainty $u_{T_{x}}$ of the temperature to be determined.

\section{Conclusion}

This paper presents a method that has been recently adopted in our laboratory to determine the temperatures of blackbody sources in the range of 1200-1570 K. The system 
is made of a Double Monochromator System (DMS) based on a grating and a prism as dispersion elements. The detection element is a silicon photodiode that is calibrated traceable to the LNE-CNAM's trap detector. In between the blackbody source and the DMS is placed the optical system consists of two convergent lenses. The crucial parameter in this study is the system responsivity " $G$ " that reflects the influence of the instrument in the temperature determination.

The optical transmission factor combined with the DMS transmission factor and the spectral sensitivity of the detector allowed us to determine the system responsivity " $G$ " of the experimental bench. The use of the DMS with a grating and a prism placed in a small area of $50 \mathrm{~cm} \times 30 \mathrm{~cm}$ compared to other comparators used by many laboratories around the world allows our system to have a fast operational readiness, high effectiveness and easy handling. Furthermore, we tried to define the responsivity of our system to stand out from the other works which they use the spectral radiance ratio of two blackbodies to trace back to the temperature.

In this work, we defined the experimental model of the system responsivity " $G$ " to be used for the temperature range between $1200 \mathrm{~K}$ and $1570 \mathrm{~K}$ and from $800 \mathrm{~nm}$ to $900 \mathrm{~nm}$. This model helps us to determine the temperature $T_{x}$ of any blackbody in this range.

The performance of the experimental bench has been thoroughly investigated considering all sources of relative uncertainty involved of the system responsivity " $G$ " which varied from $0.3 \%$ to $1.12 \%$. This in turn resulted in a corresponding uncertainty in temperature of about $2.2 \mathrm{~K}$ and $4.5 \mathrm{~K}(k=1)$ from $800 \mathrm{~nm}$ to $900 \mathrm{~nm}$.

This research and innovation are carried out under the MOBIDOC device funded by the European Union under the PASRI program administered by the ANPR.

The authors wish to thank the National Agency of Metrology Tunisia (ANM) for supporting this research.

\section{References}

1. D.C. Ripple, R. Davis, B. Fellmuth, J. Fischer, G. Machin, T. Quinn, P. Steur, O. Tamura, D.R. White, The roles of the mise en pratique for the definition of the kelvin, Int. J. Thermophys. 31, 1795-1808 (2010), doi:10.1007/s10765-010-0837-2

2. B. Fellmuth, J. Fischer, G. Machin, S. Picard, P.P.M. Steur, O. Tamura, D.R. White, H. Yoon, The kelvin redefinition and its mise en pratique, Philos. Trans. R. Soc. A 374, 20150037 (2016), doi:10.1098/rsta.2015.0037

3. E.R. Woolliams, K. Anhalt, M. Ballico, P. Bloembergen, F. Bourson, S. Briaudeau, J. Sasajima, D.R. Taubert, A.D.W. Todd, R. Van den Bossche, E. van der Ham, T. Wang, A. Whittam, B. Wilthan, D.J. Woods, J.T. Woodward, Y.
Yamada, Y. Yamaguchi, H.W. Yoon, Z. Yuan Campos, M.G. Cox, D. del Campo, W. Dong, M.R. Dury, V. Gavrilov, I. Grigoryeva, M.L. Hernanz, F. Jahan, B. Khlevnoy, V. Khromchenko, D.H. Lowe, X. Lu, G. Machin, J.M. Mantilla, M.J. Martin, H.C. McEvoy, B. Rougié, M. Sadli, S.G.R. Salim, N. Sasajima, D.R. Taubert, A.D.W. Todd, R. Van den Bossche, E. van der Ham, T. Wang, A. Whittam, B. Wilthan, D.J. Woods, J.T. Woodward, Y. Yamada, Y. Yamaguchi, H. W. Yoon, Z. Yuan, Thermodynamic temperature assignment to the point of inflection of the melting curve of high temperature fixed points, Philos. Trans. R. Soc. A 374, 20150044 (2016)

4. Y. Yamada, H. Sakate, F. Sakuma, A. Ono, Radiometric observation of melting and freezing plateaus for a series of metal-carbon eutectic points in the range $1330^{\circ} \mathrm{C}$ to $1950{ }^{\circ} \mathrm{C}$, Metrologia 36, 207-209 (1999)

5. S.G.R. Salim, K. Anhalt, D.R. Taubert, J. Hollandt, Threeelement trap filter radiometer based on large active area silicon photodiodes, Appl. Opt. 55 (15), 3958-3965 (2016)

6. E. Woolliams, G. Machin, D. Lowe, R. Winkler, Metal (carbide) - carbon eutectics for thermometry and radiometry: a review of the first seven years, Metrologia 43, R11-R25 (2006)

7. G. Machin, Twelve years of high temperature fixed point research: a review, in Temperature: its measurement and control in science and industry, Proceedings of the ninth international temperature symposium, California, pp. 305-315 (2013)

8. G. Machin, M. Sadli, R. Gavioso, J. Engert, E.R. Woolliams, The Euramet Metrology Research Programme project: implementing the new kelvin (Ink), Int. J. Thermophys. 35, 405-416 (2014), doi:10.1007/s10765-014-1606-4

9. J. Fischer, Low uncertainty Boltzmann constant determinations and the kelvin redefinition, Philos. Trans. R. Soc. A 374, 20150038 (2016), doi:10.1098/rsta.2015.0038

10. B. Khlevnoy, V. Sapritsky, B. Rougie, C. Gibson, H. Yoon, A. Gaertner, D. Taubert, J. Hartmann, CCPR-S1 supplementary comparison for spectral radiance in the range of $220 \mathrm{~nm}$ to $2500 \mathrm{~nm}$, Metrologia 46, S174-S180 (2009)

11. BIPM, Supplementary Information for the ITS 90 (2013), Chap. 6

12. G. Negro, Le corps noir. Étude et réalisation de cavités étalons de référence pour le rayonnement thermique, $\mathrm{PhD}$ thesis, École centrale Paris, 1993

13. I.E.C., Vocabulary Electrotechnical International Genève BCCI (2016), Chap. 845, Publi 50

14. J. Sacadura, Initiation aux Transferts Thermiques, 4th edn., Tec Doc (1993)

15. BOUHNIK SAS Banc d'étalonnage EIT 90 Datasheet

16. SOPRA SAS Double monochromator DMS Datasheet

17. Heitronics INFRARED RADIATION PYROMETER KT 19 II Datasheet

18. G. Asch et al., Les capteurs en instrumentation industrielle, 5th edn., Dunod (1999)

Cite this article as: Saif Abbane, Zahra Ben Achour, Oualid Touayar, Study of the system responsivity to measure the blackbody's temperature by optical pyrometry from $1200 \mathrm{~K}$ to $1570 \mathrm{~K}$, Int. J. Metrol. Qual. Eng. 8, 2 (2017) 